\title{
EMPRENDEDORISMO TECNOLÓGICO Y GÉNERO EN LA ARGENTINA: FACTORES DETERMINANTES EN LA PERCEPCIÓN DE AUTO-EFICACIA EMPRENDEDORA*
}

\author{
LUISA DE LOS ANGELES MAYORAL** \& CARMEN MARÍA SALVADOR FERRER *** \\ UNIVERSIDAD NACIONAL DEL CENTRO DE LA PROVINCIA DE BUENOS AIRES - UNICEN (ARGENTINA) \\ UNIVERSIDAD DE ALMERÍA (ESPAÑA)
}

Recibido/ Received/ Recebido: 01/12/2013 - Aceptado/ Accepted/ Aprovado: 29/05/2014

\begin{abstract}
Resumen
Este artículo es continuación de la línea de trabajo desarrollada por las autoras acerca de la existencia de diferencias de género en la relación entre tres variables centrales del emprendedorismo: autoeficacia emprendedora, inteligencia emocional y satisfacción vital. El objetivo del presente trabajo es establecer si las variables psico-sociales (inteligencia emocional y satisfacción vital) influyen en las dimensiones que conforman la auto-eficacia emprendedora de los hombres y las mujeres argentinos en el sector de las TIC. Se realizó una investigación exploratorio-descriptiva y transversal, con una muestra de 329 emprendedores, utilizando encuestas on-line. Los datos obtenidos fueron procesados y sometidos a análisis de regresión, surgiendo notables diferencias entre hombres y mujeres, especialmente en la variable satisfacción vital. Particularmente, en el caso de los hombres se observa que la inteligencia emocional es determinante para desarrollar nuevos productos y oportunidades de mercado, mientras que la inteligencia emocional y la satisfacción vital influyen en el resto de las dimensiones (gestión de recursos humanos, nuevas relaciones con inversores, creación de entornos innovadores y trabajo bajo estrés). Estos hallazgos difieren en el caso de las mujeres, donde se aprecia que sólo la inteligencia emocional influye en la totalidad de las dimensiones que conforman la auto-eficacia emprendedora.
\end{abstract}

Palabras clave: Auto-eficacia emprendedora, Inteligencia emocional, Satisfacción vital, Género, TIC.

\section{TECHNOLOGICAL AND GENDER ENTREPRENEURISM IN ARGENTINA: DETERMINANT FACTORS ON THE PERCEPTION ENTERPRISING SELF-EFFICACY}

\begin{abstract}
This article is the continuation of the work developed by the authors about the existence of gender differences in relationship between three key variables of entrepreneurism: enterprising self-efficacy,
\end{abstract}

\footnotetext{
Artículo de investigación derivado del proyecto titulado "Normas vigentes en el ámbito laboral". Se desarrolló en el periodo 2011 2013. Financiado por la Universidad Nacional del Centro de la Provincia de Buenos Aires - UNICEN.

** Profesora Titular de la Universidad Nacional del Centro de la Provincia de Buenos Aires - UNICEN (Argentina). Correo electrónico: mayoral.luisa@gmail.com. Tel: 54294 4439550. Dirección postal: UNICEN, Facultad de Ciencias Económicas, Centro de Estudios en Administración. Campus Universitario. Paraje Arroyo Seco s/n. C.P. 7000, Tandil.

**** Profesora Titular de la Universidad de Almería (España). Correo electrónico: cmsalva@ual.es. Tel: 950014047. Dirección postal: Universidad de Almería, Facultad de Psicología. Carretera de Sacramento. La Cañada de San Urbano, s/n. C.P. 04120, Almería.
} 
emotional intelligence and life satisfaction. The aim of this study is to establish whether psychosocial variables (emotional intelligence and life satisfaction) influence dimensions that make enterprising self-efficacy of Argentine men and women in the ICT sector. An exploratory-descriptive and cross-sectional survey was conducted with a sample of 329 entrepreneurism, using on-line surveys. The data obtained were processed and analyzed through regression analysis, significant differences emerged between men and women, especially in life satisfaction variable. Particularly men case shows that emotional intelligence is crucial to develop new products and market opportunities, while emotional intelligence and life satisfaction influence the other dimensions (human resource management, new relationships with investors, creating innovative work environments and under stress). These findings differ in women case, it was observed that only emotional intelligence affects all the dimensions which constitute enterprising self-efficacy.

Keywords: Enterprising self-efficacy, Emotional intelligence, Vital satisfaction, Gender, ICT.

\title{
EMPREENDEDORISMO TECNOLÓGICO E GÊNERO NA ARGENTINA: FATORES DETERMINANTES NA PERCEPÇÃO DE AUTOEFICÁCIA EMPREENDEDORA
}

\begin{abstract}
Resumo
Este artigo é a continuação da linha de trabalho desenvolvida pelas autoras a respeito da existência de diferenças de gênero na relação entre três variáveis centrais do empreendedorismo: autoeficácia empreendedora, inteligência emocional e satisfação vital. O objetivo do presente trabalho é estabelecer se as variáveis psicossociais (inteligência emocional e satisfação vital) influenciam nas dimensões que formam a autoeficácia empreendedora dos homens e mulheres argentinos no setor das TIC. Realizou-se uma pesquisa exploratório-descritiva e transversal, com uma mostra de 329 empreendedores, utilizando-se questionários on-line. Os dados obtidos foram processados e submetidos a análises de regressão, surgindo notáveis diferenças entre homens e mulheres, especialmente na variável satisfação vital. Particularmente, no caso dos homens se observa que a inteligência emocional é determinante para desenvolver novos produtos e oportunidades de mercado, enquanto a inteligência emocional e a satisfação vital influenciam nas outras dimensões (gestão de recursos humanos, novas relações com investidores, criação de meios inovadores e trabalho sob pressão). Estes resultados diferem do caso das mulheres, onde se vê que só a inteligência emocional influencia na totalidade das dimensões que formam a autoeficácia emprendedora.

Palavras chave: Autoeficácia empreendedora, Inteligência emocional, Satisfação vital, Gênero, TIC.

Mayoral, L. \& Salvador, C. (2014) Emprendedorismo tecnológico y género en la Argentina: factores determinantes en la percepción de auto-eficacia emprendedora. En: Revista de la Facultad de Ciencias Económicas de la Universidad Militar Nueva Granada. rev.fac.cienc.econ, XXII (2).
\end{abstract}

JEL: O31, O32, O39.

\section{Introducción}

El emprendedorismo como fenómeno global está siendo asociado en forma creciente al avance tecnológico, no obstante de lo cual, aún se sabe poco de la naturaleza del fenómeno emprendedor en el sector TIC. Tradicionalmente, se ha considerado al emprendedor como un individuo cuyos comportamientos están determinados por características personales e intrínsecas antes que por factores externos. Sin 
embargo, no existe un acuerdo sobre cuáles son los rasgos de personalidad propios de estos emprendedores (Chell, Haworth \& Brearley, 1991), así como tampoco está claro cuánto hay de personal y cuánto de contexto en dicha dinámica emprendedora.

Un reflejo de toda esta diversidad de opiniones puede observarse en los programas de formación de emprendedores, donde bajo el mismo título suelen agruparse miradas muy distintas sobre un mismo tema. Desde ejercicios de auto-estima hasta planes de negocios, parece que todo es posible y va a cuento en el desarrollo del emprendedorismo. Se sabe tan poco aún, que cualquier intento bien intencionado parece suponer una aportación científica. Ahora bien, si se coincide con Bandura (1997, 1999), está claro que cualquier iniciativa tendiente a fomentar el emprendedorismo debería incorporar el desarrolo de una capacidad emprendedora auto-percibida alta, fuerte y funcional. No obstante, al igual que ocurre en otras áreas de aplicación de la auto-eficacia, en la investigación de la conducta emprendedora existen dos aproximaciones diferentes (Moriano, Palaci \& Morales, 2006). En un caso, se entiende la auto-eficacia en un sentido amplio y general, como la creencia o confianza en la propia habilidad para rendir exitosamente (Markman, Baron \& Balkin, 2005), mientras que desde otra vertiente, los estudios se centran en el desarrollo de escalas de autoeficacia específicas al dominio de los emprendedores (Chen, Green \& Crick, 1998).

Finalmente, es importante destacar que el sector TIC en Argentina evidencia un cierto dinamismo en los últimos años. Sin embargo, la formación de emprendedores $-y$ por ende, de emprendedores del sector TIC- es hoy motivo de estudio, ya que involucra una multiplicidad de conceptos que, en general, no se aprenden en entornos educativos convencionales $y$ generan déficits o áreas de debilidad en la propia formación empresarial (Kantis, Angelelli \& Moori Koenig, 2004; Kantis, Federico \& Drucaroff, 2005).

No obstante, aún queda mucho trabajo que realizar, siendo especialmente relevante conocer la percepción que hombres y mujeres tienen de la auto-eficacia emprendedora, ya que tal como reflejan algunos autores (Minniti, Arenius \& Langowitz, 2005; Mue- ller \& Conway, 2013) parece que existe una brecha entre hombres y mujeres emprendedores, siendo, asimismo, interesante conocer la relación con otras variables de naturaleza psicológica (Kickul, Gundry, Barbosa \& Whitcanack, 2009). En una investigación anterior, Salvador, Mayoral \& Errandoroso (2012) encontraron diferencias de género en los emprendedores argentinos en el sector TIC, así como también pudo determinarse, en aquella oportunidad, que la satisfacción vital ejercía una influencia fundamental en la auto-eficacia emprendedora masculina.

Es aquí donde se enmarca el presente estudio, el cual se constituye en una continuación y una profundización de aquel trabajo, y que a efectos de una rápida comprensión por parte del lector se presenta a continuación la Gráfica 1, la que representa el marco teórico de este artículo. En ella se ven representadas las variables dependientes y las variables independientes, así como las relaciones que serán objeto de estudio.

Tal como se observa en la Gráfica 1, dentro del presente trabajo $-\mathrm{y}$ como continuación del estudio expuesto por Salvador, Mayoral \& Errandosoro (2012) - se estudian dos variables independientes (inteligencia emocional y satisfacción vital), así como cada una de las dimensiones que conforman la auto-eficacia emprendedora, las cuales serán utilizadas como variables dependientes, en concreto, desarrollo de nuevos productos y oportunidades de mercado, gestión de recursos humanos, relaciones con nuevos inversores, construcción de un entorno innovador y trabajo bajo estrés. Por consiguiente, las preguntas que se intenta abordar con el presente estudio son si la satisfacción vital y la inteligencia emocional ejercen una influencia estadísticamente significativa sobre cada una de las dimensiones de la auto-eficacia emprendedora y si en esa relación existen diferencias de género estadísticamente significativas.

\section{Marco teórico}

\subsection{Variable dependiente: auto-eficacia emprendedora}

El concepto de auto-eficacia remite a la creencia en las propias capacidades y habilidades para iniciar 
Gráfica 1. Representación gráfica del presente trabajo.

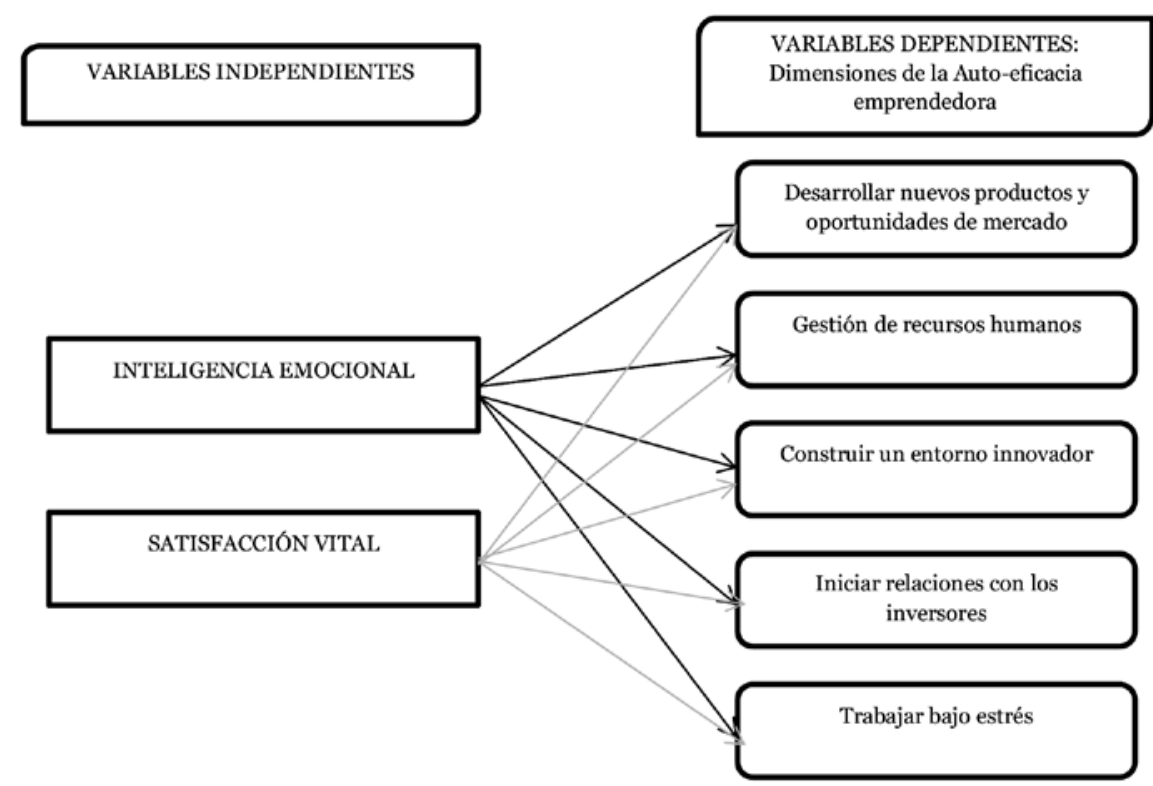

actividades y llevarlas a cabo en forma exitosa (Bandura, 1997; Salvador \& Morales, 2009). En este sentido, se considera que la capacidad para la auto-reflexión es la capacidad más singularmente humana, por esta forma de auto-referencia del pensamiento que permite evaluar y alterar tanto el propio pensamiento como la conducta (Bandura, 1987). Finalmente, estas evaluaciones incluyen percepciones de auto-eficacia, que es como Bandura $(1999,3)$ define a "las creencias en las capacidades propias para organizar y ejecutar los cursos de acción requeridos que producirán determinados logros o resultados".

En cualquier caso, lo que sí queda claro es que la auto-eficacia percibida afecta el comportamiento no sólo directamente, sino también como variable interviniente y mediadora, condicionando aspiraciones, expectativas, predisposiciones y hasta la evaluación de posibilidades de éxito/fracaso en el medio social (Bandura, 1997).

Hace ya unos años, ha surgido el constructo de auto-eficacia emprendedora (De Noble, Jung \& Lich, 1999), en relación a la creencia de las personas en sus propias habilidades para llevar a cabo las distintas tareas implicadas en la creación de una nueva empresa. Así, se ha podido determinar que la autoeficacia emprendedora constituye un atributo distintivo de los emprendedores en comparación con otros grupos (Markman et al., 2005).

En esta línea, Moriano et al. (2006) realizaron un trabajo donde validaron una escala de auto-eficacia emprendedora, mostrando que este constructo se compone de cinco dimensiones, las cuales serán utilizadas como variables dependientes en el presente estudio. En concreto, tal como citan Moriano et al. (2006), las dimensiones son las siguientes:

- Desarrollar nuevos productos y oportunidades de mercado. Se trata de la capacidad que tiene el emprendedor para desarrollar sus productos y adaptarse al mercado.

- Gestionar los recursos humanos. Referido a la capacidad que permite al emprendedor atraer y retener a personas clave en la empresa.

- Construir un entorno innovador. Entendido como la capacidad de construir un entorno propicio a la innovación, en base a la creatividad, la iniciativa y la responsabilidad. 
- Iniciar relaciones con los inversores. Alude a la capacidad para usar las redes y establecer contactos en beneficio de la empresa.

- Trabajar bajo estrés. Se trata de la capacidad que tiene el emprendedor de trabajar en situaciones de incertidumbre, cambio y estrés.

En síntesis, a lo largo del presente artículo se utilizarán estas cinco dimensiones como variables dependientes. En concreto, el interés reside en explorar la influencia que determinadas variables psicosociales (satisfacción vital e inteligencia emocional) ejercen en cada uno de los componentes de esta auto-eficacia emprendedora.

\subsection{Variable independiente: satisfacción vital}

El concepto de satisfacción vital como componente cognitivo del bienestar subjetivo es entendido en relación a la valoración positiva que cada persona hace de su vida en general, o de aspectos particulares de esta -familia, estudios, trabajo, salud, amigos, tiempo libre- (Diener, 1994; García, 2002; Diener, Suh, Lucas \& Smith, 1999, García-Viniegras \& González, 2000; Liberalesso, 2002), siendo los dominios de funcionamiento más cercanos e inmediatos a la vida personal de los individuos los que tienen mayor influencia sobre este bienestar. Así, las personas evalúan su estado actual con las expectativas de futuro que tienen para sí mismas (Moyano \& Ramos, 2007), siendo la satisfacción la diferencia percibida entre sus aspiraciones y sus logros.

De acuerdo a Salvador \& Morales (2009), en los últimos treinta años se ha incrementado considerablemente el número de investigaciones relacionadas con los diferentes aspectos del bienestar subjetivo (Diener, 1984). Los primeros trabajos en este sentido, se centraban en analizar el papel que desempeñaban las variables demográficas en la satisfacción vital depositando el interés en variables "externas" al individuo. Contrariamente, la tendencia actual en la investigación psicológica del bienestar subjetivo ha recuperado el análisis de las variables "internas".

Durante las tres últimas décadas esta tendencia ha quedado reflejada en las siguientes tres posturas teó- ricas (Díaz \& Sánchez, 2002). La primera, asume que la satisfacción con la vida en general depende de la suma de las satisfacciones en cada uno de los dominios de vida (teoría botton-up); la segunda, postula que la satisfacción con la vida en general determina el nivel de satisfacción en cada una de las áreas vitales (teoría top-down). La tercera perspectiva teórica ha sido consecuencia de la falta de resultados consistentes en cada una de las anteriores y asume que la relación es bidireccional.

Parece lógico pensar que la satisfacción con un aspecto de la vida debería tener implicaciones para la satisfacción con otros aspectos (Salvador \& Morales, $2009,2013)$. Actualmente y respecto de este particular, según recogen Salvador \& Mayoral (2009, 2013), existen dos posturas confrontadas. Una de ellas es la hipótesis del desbordamiento que sugiere que una alta satisfacción o insatisfacción en uno de los aspectos puede desbordarse hacia el otro, dando lugar a la misma actitud -relación positiva entre los dos tipos de satisfacción-. En el sentido opuesto, se encuentra la hipótesis de la compensación donde se defiende que una alta satisfacción en un dominio (por ejemplo, "trabajo") puede compensar la menor satisfacción en otros dominios (por ejemplo, "no trabajo"), o a la inversa -relación negativa entre los dos tipos de satisfacción-.

En cualquier caso, en lo que sí existe acuerdo es en que la satisfacción vital podría entenderse como la evaluación global que hace una persona de su vida (Atienza, Pons, Balaguer \& García- Merita, 2000; Pavot, Diener, Colvin \& Sandvik, 1991). En otras palabras, el individuo examina los aspectos tangibles de su vida, sopesa lo bueno contra lo malo, lo compara con un estándar o criterio elegido por ella (Shin \& Johnson, 1978) y llega a un juicio sobre su satisfacción (Pavot et al., 1991).

En el mismo sentido, Diener, Emmons, Larsen \& Griffin, (1985) afirman que las investigaciones sobre la satisfacción vital deberían centrarse en analizar los juicios subjetivos que hace la persona sobre su vida; esto es, en lugar de sumar la satisfacción a través de dominios específicos, sería recomendable y necesario preguntar a la persona por la evaluación global de su vida (Diener et al., 1985). Esta evaluación de 
tipo subjetiva es la que se ha solicitado que realicen emprendedores y emprendedoras del sector TIC, a los efectos del presente estudio.

\subsection{Variable independiente: inteligencia emocional}

En la última década la investigación sobre regulación y manejo de las emociones ha experimentado un gran interés (Fernández-Berrocal \& Extremera, 2006; Rego \& Fernández, 2005; Salovey, Mayer, Goldman, Turvey \& Palfai, 1995). Este esplendor se debe en gran parte al surgimiento de un nuevo concepto denominado Inteligencia emocional -IE- definido como "una parte de la inteligencia social que incluye la capacidad de controlar nuestras emociones y las de los demás, discriminar entre ellas y usar dicha información para guiar nuestro pensamiento y nuestros comportamientos" (Salovey \& Mayer, 1990, 239).

Por tanto, la aptitud emocional mide la capacidad de comprender, procesar, controlar y expresar aspectos emocionales y sociales de nuestra vida (Mayer \& Salovey, 1997). El grado en que somos capaces de hacerlo determina la productividad y la satisfacción que se obtienen en la vida, mientras que el nivel y la calificación de estudios académicos por sí mismos no lo determinan (Goleman, 1995; Heath, 1991; Salvador, 2012a, 2012b). Esto significa, según señala Cohen $(2003,21)$ que "la aptitud emocional se refiere a la capacidad de comprender y expresar los aspectos emocionales y sociales de nuestra vida". Finalmente, es esta inteligencia o dominio emocional, de naturaleza compleja, aunque parece ser un término de sumo interés ha sido poco estudiada en lo que respecta la relación con la auto-eficacia emprendedora. Por ende, en esta investigación se empleará este término (inteligencia emocional) como segunda variable independiente del presente estudio.

Como se ha apuntado previamente, este estudio es una continuación del trabajo presentado por Salvador, Mayoral \& Errandoroso (2012). En aquel trabajo, los autores encontraron diferencias de género en los emprendedores argentinos en el sector de las TIC, observando asimismo que la satisfacción vital ejercía una influencia fundamental en la auto-efica- cia emprendedora de los hombres emprendedores. Así, pues, el presente trabajo constituye una profundización del anterior, al procurar analizar la influencia de la inteligencia emocional y la satisfacción vital en cada una de las dimensiones que, según Moriano et al. (2006) conforman la escala de auto-eficacia emprendedora -desarrollo de nuevos productos y oportunidades de mercado, gestión de recursos humanos, creación de entorno innovador, nuevas relaciones y trabajo bajo estrés-.

\section{Método}

\subsection{Procedimiento}

El trabajo de investigación que se presenta es de carácter exploratorio-descriptivo y transversal. Este se ha llevado a cabo en todas las organizaciones, privadas y públicas que agrupaban emprendedores tecnológicos en la Argentina, al momento de la toma de los datos primarios, y conforme a una búsqueda exhaustiva en la red, dada la carencia de bases de datos actualizadas a nivel nacional.

Una vez realizada una primera detección, se contactó a los representantes de cada una de estas instituciones (polos tecnológicos, asociaciones de emprendedores tecnológicos, cámaras de emprendedores de software, organizaciones financiadoras de proyectos de naturaleza tecnológica, organismos públicos con concursos regulares de fondos para el financiamiento de emprendedores tecnológicos, etc.) para solicitarles el apoyo y difusión a la investigación que iba a realizarse, así como, también, pedirles información acerca de otras instituciones que no hubieran sido detectadas en una primera exploración. Por otro lado, se indagó muy activamente acerca de la existencia de emprendedores "aislados" a partir de la exploración de las listas de participantes en las convocatorias a premios a la innovación y al emprendedorismo tecnológico de naturaleza tanto privada como pública en los últimos cinco años, al momento del relevamiento (fecha coincidente con el registro en la red de aquellos primeros premios).

Finalmente, por cuanto los emprendedores tecnológicos suelen conocerse unos a otros, se utilizó la técnica de "bola de nieve", para que una vez detectado 
un emprendedor, éste señalara a otros colegas, llegando a una fase de saturación, en que los nombres se repetían insistentemente.

Habiendo llegado a la detección de un número de 949 emprendedores, reunidos en su mayoría en asociaciones y radicados en las ciudades de Buenos Aires, La Plata, Rosario, Tandil, Córdoba, Mar de la Plata y Bahía Blanca, se procedió a subir la encuesta a la red por un plazo de tres meses (abril, mayo y junio de 2010), previo contacto vía correo electrónico, indagando acerca de la predisposición del emprendedor para responderla. Este procedimiento de progresivo acercamiento y detección, así como de cautela en la obtención de un compromiso de responder la encuesta, sumado al hecho de que ésta fuera virtual $y$, en consecuencia, susceptible de ser completada en cualquier lugar y tiempo dentro de los tres meses, hizo que los emprendedores no mostraran resistencias importantes, a la vez que comprometió a los investigadores a una devolución de resultados, actividad que se realizó en una etapa posterior.

Finalmente, cumplido el término estipulado la encuesta se bajó de la red y se procedió al procesamiento y análisis de resultados, en el paquete estadístico SPSS (versión 17 para Windows). Solo se consideraron las encuestas que fueron respondidas en su totalidad, número que ascendió a 329 (35\% del total).

\subsection{Muestra}

La muestra total se conforma de 329 emprendedores argentinos y argentinas, del sector TIC, de los cuales $69 \%$ son hombres y $31 \%$ son mujeres. Las edades de los mismos se encuentran comprendidas entre los 15 y los 68 años, siendo 27 años la edad mayoritaria.

\subsection{Instrumento}

Respecto del instrumento utilizado, se exponen las distintas escalas utilizadas en el trabajo de campo y las principales características psicométricas de las mismas:

- Auto-eficacia-emprendedora: La herramienta utilizada es la Entreprenuerial Self-Efficacy
(ESE) de Noble et al. (1999) en su versión en castellano (Moriano et al., 2006). En este sentido, tras someter el instrumento a un proceso de validación, la edición final contiene 23 items con formato tipo Likert de 5 puntos ( 1 = "completamente incapaz" y $5=$ "perfectamente capaz"). Los resultados obtenidos en el coeficiente de fiabilidad son de 0,93.

- Satisfacción vital: La herramienta utilizada para medir la satisfacción vital deriva de Diener et al. (1985, 1999). Se trata de una escala con cinco preguntas con formato tipo Likert de 5 puntos donde el valor 1 significa "muy de acuerdo" y el valor 5 , todo lo contrario. El alfa de Cronbach obtenido para esta escala es de 0,802.

- Inteligencia emocional: El instrumento utilizado ha sido el diseñado por Salvador (2010). Se trata de un cuestionario tipo Likert de 5 puntos donde el valor 1 representa el máximo desacuerdo con lo preguntado, mientras que el valor 5 refleja todo lo contrario. La puntuación obtenida en la prueba alfa de Cronbach asciende a 0,64 .

\section{Resultados}

Con el fin de analizar los datos, se trabajó con modelos de regresión (método enter). Así, pues, se utilizaron las variables independientes inteligencia emocional y satisfacción vital, mientras que las variables dependientes se conforman con las distintas dimensiones de la auto-eficacia emprendedora, explorando asimismo la presencia de diferencias de género. Por ello, a continuación se exponen los datos referidos a los hombres y mujeres, detallando las cinco dimensiones de la auto-eficacia emprendedora como variables dependientes -desarrollar nuevos productos y oportunidades de mercado, gestionar recursos humanos, iniciar nuevas relaciones con inversores, construir un entorno innovador y trabajar bajo estrés-.

En el caso de los hombres, se observa que en lo que respecta al "desarrollo de nuevos productos y oportunidades de mercado" (Tabla 1), los datos generales son estadísticamente significativos (Rcua- 
drado corregido 0,80, F=10,81 y Sig. $=0,000$ ). En el análisis detallado, se aprecia que la inteligencia emocional influye sobre el desarrollo de nuevos productos y oportunidades de mercado (Beta $=0,252$; Sig. $=0,000)$. Finalmente, la satisfacción vital no ejerce ninguna influencia destacable.

Tabla 1. Análisis de regresión de la variable dependiente: desarrollo de nuevos productos y oportunidades de mercado $^{1}$

\begin{tabular}{|c|c|c|c|c|c|}
\hline & B & Error típ. & Beta & T & Sig. \\
\hline IE & 0,456 & 0,119 & 0,252 & 3,828 & 0,000 \\
\hline SVITAL & 0,095 & 0,057 & 0,111 & 1,682 & 0,094 \\
\hline
\end{tabular}

En la dimensión "Gestión de Recursos humanos" (Tabla 2), los datos generales son estadísticamente significativos (Rcuadrado corregido 0,116, $\mathrm{F}=15,82$ y Sig. $=0,000$ ). En lo que respecta al análisis particular, los datos incluidos en la Tabla 2 reflejan que, en el caso de los hombres, en la gestión de recursos humanos influye tanto la inteligencia emocional (Beta $=0,273 ;$ Sig. $=0,000)$ como la satisfacción vital $($ Beta $=0,169 ;$ Sig. $=0,009)$.

Tabla 2. Análisis de regresión de la variable dependiente: gestión de recursos humanos²

\begin{tabular}{|c|c|c|c|c|c|}
\hline & B & Error típ. & Beta & T & Sig. \\
\hline IE & 0,509 & 0,120 & 0,273 & 4,236 & 0,000 \\
\hline SVITAL & 0,150 & 0,057 & 0,169 & 2,619 & 0,009 \\
\hline
\end{tabular}

En lo que respecta a la dimensión que alude a "iniciar relaciones con los inversores" (Tabla 3), se observa que los datos son significativos (Rcuadrado corregido $0,135, \mathrm{~F}=18,63$ y Sig. $=0,000$ ). En el análisis detallado se encuentra que en el caso de los hombres, a la hora de iniciar relaciones con los inversores es importante tanto la inteligencia emocional (Beta $=0,255$; Sig. $=0,000)$ como la satisfacción vital $($ Beta $=0,226$; Sig. $=0,000$ ). En otras palabras, para que los hombres inicien relaciones con los inversores es preciso que tengan dominio emocional y satisfacción vital.

Tabla 3. Análisis de regresión de la variable dependiente: iniciar relaciones con los inversores ${ }^{3}$

\begin{tabular}{|c|c|c|c|c|c|}
\hline & B & Error típ. & Beta & T & Sig. \\
\hline IE & 0,474 & 0,118 & 0,255 & 4,007 & 0,000 \\
\hline SVITAL & 0,200 & 0,056 & 0,226 & 3,550 & 0,000 \\
\hline
\end{tabular}

Por otro lado, cuando se estudia la dimensión "construir un entorno innovador" (Tabla 4) se observa que los datos son estadísticamente significativos (Rcuadrado corregido $0,185, F=26,50$ y Sig. $=0,000)$. En el análisis detallado puede apreciarse que sobre esta variable dependiente influye tanto la inteligencia emocional (Beta $=0,394$; Sig. $=0,000)$ como la satisfacción vital $($ Beta $=0,122 ;$ Sig. $=0,05)$.

Tabla 4. Análisis de regresión de la variable dependiente: construir un entorno innovador ${ }^{4}$

\begin{tabular}{|c|c|c|c|c|c|}
\hline & B & Error típ. & Beta & T & Sig. \\
\hline IE & 0,749 & 0,118 & 0,394 & 6,360 & 0,000 \\
\hline SVITAL & 0,110 & 0,056 & 0,122 & 1,973 & 0,050 \\
\hline
\end{tabular}

Cuando se estudia la dimensión "trabajar bajo estrés" (Tabla 5), se descubre que los datos generales pertenecientes al análisis de regresión son estadísticamente significativos (Rcuadrado corregido $0,063, F=8,60$ y Sig. $=0,000$ ). En el análisis particular, los datos incluidos en la Tabla 5 indican que, en el caso de los hombres, la inteligencia emocional $($ Beta $=0,150 ;$ Sig. $=0,024)$ y la satisfacción vital (Beta $=0,189$; Sig. $=0,024)$ son importantes para trabajar bajo estrés. No obstante, hay que destacar que el valor global es muy bajo, es decir, aunque estas variables (inteligencia emocional y satisfacción vital) influyen en esta variable de la auto-eficacia emprendedora, su impacto es menor que otras dimensiones de la auto-eficacia.

Fuente: Elaboración propia.

Fuente: Elaboración propia.

Fuente: Elaboración propia.

Fuente: Elaboración propia. 
Tabla 5. Análisis de regresión de la variable dependiente: trabajar bajo estrés ${ }^{5}$

\begin{tabular}{|c|c|c|c|c|c|}
\hline & B & Error típ. & Beta & T & Sig. \\
\hline IE & 0,323 & 0,143 & 0,150 & 2,266 & 0,024 \\
\hline SVITAL & 0,194 & 0,068 & 0,189 & 2,853 & 0,005 \\
\hline
\end{tabular}

A continuación, los datos obtenidos en el caso de las mujeres. En la Tabla 6, correspondiente a la dimensión "desarrollo de nuevos productos y oportunidades de mercado", los datos generales son estadísticamente significativos (Rcuadrado corregido 0,262, $\mathrm{F}=19,090$ y Sig. =0,000). En el análisis detallado, se observa que el dominio emocional influye en el desarrollo de nuevos productos y oportunidades de mercado $($ Beta $=0,528 ;$ Sig. $=0,000)$.

Tabla 6. Análisis de regresión de la variable dependiente: desarrollo de nuevos productos y oportunidades de mercado $^{6}$

\begin{tabular}{|c|c|c|c|c|c|}
\hline & B & Error típ. & Beta & T & Sig. \\
\hline IE & 1,181 & 0,191 & 0,528 & 6,176 & 0,000 \\
\hline SVITAL & $-0,062$ & 0,083 & $-0,064$ & $-0,745$ & 0,458 \\
\hline
\end{tabular}

Tal como reflejan los datos (Tabla 7), correspondientes a la variable dependiente "gestión de recursos humanos" de las mujeres emprendedoras argentinas (Rcuadrado corregido $0,304, F=23,22$ y Sig. $=0,000$ ) los resultados son estadísticamente significativos, siendo la inteligencia emocional (Beta $=0,559$; Sig. $=0,000$ ) el único constructo determinante de la dimensión gestión de recursos humanos.

Tabla 7. Análisis de regresión de la variable dependiente: gestión de recursos humanos ${ }^{7}$

\begin{tabular}{|c|c|c|c|c|c|}
\hline & B & Error típ. & Beta & T & Sig. \\
\hline IE & 1,282 & 0,190 & 0,559 & 6,739 & 0,000 \\
\hline SVITAL & 0,033 & 0,083 & 0,033 & 0,399 & 0,691 \\
\hline
\end{tabular}

En la tabla 8 se reflejan los datos pertenecientes a las mujeres en lo que respecta a "iniciar relaciones con los inversores" (Rcuadrado corregido 0,228, F=16,10 y Sig. $=0,000)$. De manera detallada, se aprecia que la inteligencia emocional influye en las relaciones con los inversores (Beta $=0,495$; Sig. $=0,000)$, no sucediendo lo mismo con la satisfacción vital.

Tabla 8. Análisis de regresión de la variable dependiente: iniciar relaciones con los inversores ${ }^{8}$

\begin{tabular}{|c|c|c|c|c|c|}
\hline & B & Error típ. & Beta & T & Sig. \\
\hline IE & 1,042 & 0,184 & 0,495 & 5,671 & 0,000 \\
\hline SVITAL & $-0,023$ & 0,080 & $-0,025$ & $-0,283$ & 0,778 \\
\hline
\end{tabular}

Cuando se estudia la dimensión de la auto-eficacia emprendedora definida como "construir un entorno innovador" (Tabla 9), se observa la significatividad de los datos generales (Rcuadrado corregido 0,269, $\mathrm{F}=19,78$ y Sig. $=0,000)$. Así, pues, en el análisis detallado, los datos pertenecientes a las mujeres reflejan que en esta dimensión influye la inteligencia emocional (Beta=0,532; Sig. $=0,000)$.

Tabla 9. Análisis de regresión de la variable dependiente: construir un entorno innovador ${ }^{9}$

\begin{tabular}{|c|c|c|c|c|c|}
\hline & B & Error típ. & Beta & T & Sig. \\
\hline IE & 1.146 & .183 & .532 & 6.261 & .000 \\
\hline SVITAL & .003 & .079 & .003 & .034 & .973 \\
\hline
\end{tabular}

Finalmente, en el caso de las mujeres (Tabla 10) los datos generales pertenecientes a "trabajar bajo estrés", son estadísticamente significativos (Rcuadrado corregido 0,218, F=15,19 y Sig.=0,000). Del mismo modo que en los casos anteriores, se observa que sobre esta dimensión influye la inteligencia emocional (Beta=0,470; Sig. =0,000).

\footnotetext{
Fuente: Elaboración propia.

Fuente: Elaboración propia.

Fuente: Elaboración propia.

Fuente: Elaboración propia.

9 Fuente: Elaboración propia.
} 
Tabla 10. Análisis de regresión de la variable dependiente: trabajar bajo estrés ${ }^{10}$

\begin{tabular}{|c|c|c|c|c|c|}
\hline & B & Error típ. & Beta & T & Sig. \\
\hline IE & 1,169 & 0,219 & 0,470 & 5,348 & 0,000 \\
\hline SVITAL & 0,080 & 0,095 & 0,074 & 0,843 & 0,401 \\
\hline
\end{tabular}

En síntesis, los datos indican que respecto de las variables analizadas, los emprendedores argentinos tienen perfiles diferentes, según sean hombres o mujeres. En concreto, los datos indican que los hombres necesitan tener satisfacción vital para sentir que pueden tener éxito en el emprendimiento. No sucede lo mismo con las mujeres, las cuales no necesitan tener satisfacción vital para sentirse con capacidad de emprender exitosamente. No obstante, hay un punto en el que convergen hombres y mujeres y es que ambos entienden que la inteligencia emocional es importante para emprender.

A continuación, es importante detenerse en un análisis más detallado de cómo influyen sendos términos (satisfacción vital e inteligencia emocional) en las distintas dimensiones que conforman la auto-eficacia emprendedora en los hombres y las mujeres argentinos. Así, en el caso de los hombres se observa que en el desarrollo de nuevos productos y oportunidades de mercado sólo influye la inteligencia emocional, mientras que en el resto de dimensiones (gestionar los recursos humanos, iniciar nuevas relaciones con los inversores, construir un entorno innovador y trabajar bajo estrés) es importante tanto la inteligencia emocional como la satisfacción vital. En lo concerniente a la dimensión de trabajar bajo estrés, estas variables son débiles predictores. En el caso de las mujeres, se aprecia que la inteligencia emocional influye sobre las cinco dimensiones que conforman la auto-eficacia emprendedora. Sin embargo, y al igual que se señaló respecto de los hombres, la inteligencia emocional es un predictor bajo cuando se trata de trabajar bajo estrés. Como conclusión, hombres y mujeres emprendedores argentinos consideran que en el desarrollo de nuevos productos y oportunidades de mercado es importante la inteligencia emocional, mientras que en el resto de dimensiones pueden encontrarse diferencias.

\section{Conclusiones}

Como principal conclusión de este trabajo, y tal como apuntaban Salvador \& Mayoral (2013), se resalta la existencia de diferencias de género en los emprendedores argentinos en el sector TIC. Nótese al respecto que son escasos los estudios que analizan las diferencias de género en la auto-eficacia emprendedora (Mueller \& Conway, 2013). Así, a grandes rasgos podría citarse el trabajo de Wilson, Kickul \& Marlino (2007), quienes también hallaron diferencias de género en la auto-eficacia emprendedora.

No obstante, este artículo supone una contribución para la comunidad científica, por varias razones: en primer lugar, porque muestra estas diferencias estadísticamente significativas en el sector TIC y, en segundo lugar, porque ofrece resultados más detallados, ya que la mayoría de los trabajos se centran en el análisis global de la auto-eficacia emprendedora con otras variables $y$, no como la presente investigación, en la cual se obtienen datos acerca de las relaciones entre las dimensiones que, según Moriano et al., 2006), conforman la auto-eficacia emprendedora (utilizadas aquí como variables dependientes).

En concreto, en el caso de las mujeres, se observa que la satisfacción vital no es determinante, mientras que la inteligencia emocional adquiere un peso central en las cinco dimensiones estudiadas (desarrollo de nuevos productos y oportunidades de mercado, gestión de recursos humanos, creación de entornos innovadores, relaciones con nuevos inversores y trabajo bajo estrés).

En el caso de los hombres, se observa que en cuatro de las cinco dimensiones (gestión de recursos humanos, relaciones con nuevos inversores, creación de entornos innovadores y trabajo bajo estrés) son fundamentales tanto la satisfacción vital como la inteligencia emocional. No obstante, estos datos no se verifican cuando se estudia la dimensión correspondiente al desarrollo de nuevos productos y oportunidades de mercado, donde sólo se observa el impacto de la inteligencia emocional. Esto hace suponer que para que los hombres argentinos se sientan capaces a la hora

10 Fuente: Elaboración propia. 
de emprender resulta básico que estén satisfechos con su vida y que, también, tengan dominio emocional.

De igual forma, los datos indican que, en el caso de las mujeres, la inteligencia emocional impacta muy fuertemente en la dimensión de auto-eficacia referida a la gestión de recursos humanos. Por su parte, en el caso de los hombres, el mayor peso se obtiene en la inteligencia emocional cuando impacta sobre la construcción de entornos innovadores. Por consiguiente, estos datos confirman que, a pesar de existir diferencias de género en los emprendedores argentinos, resulta básico trabajar la inteligencia emocional.

Además, es importante destacar un dato común y es el hecho de que tanto los hombres como las mujeres emprendedoras argentinas crean que la inteligencia emocional es fundamental para el desarrollo de nuevos productos y oportunidades de mercado y oportunidades de mercado. Este dato parece reforzar la necesidad de generar programas de entrenamiento en habilidades emocionales para lograr una más alta percepción de auto-eficacia en el rol emprendedor.

Cierto es que estos hallazgos se alinean, en cierto sentido con los de otros autores (Chen, Greene \& Crick, 1998; Scherer, Brodzinski \& Wiebe, 1990; Wilson, Kickul \& Marlino, 2007) que establecen la existencia de diferencias de género en la percepción de auto-eficacia emprendedora. Por otra parte, los datos obtenidos reflejan que la inteligencia emocional es un predictor de las distintas dimensiones -desarrollo de nuevos productos y oportunidades de mercado, gestión de recursos humanos, relaciones con inversores, construcción de un entorno innovador y trabajo bajo estrés- que conforman la auto-eficacia emprendedora.

En cualquier caso, tal como señalan Salvador \& Mayoral (2013), lo que se hace evidente en trabajos como este es que el factor humano es importante para conocer el perfil emprendedor. Los hallazgos obtenidos señalan que es necesario tener presente -por obvio que parezca- que las personas son personas y que para llevar adelante sus propios negocios -en este caso, en el sector TIC argentino-, debe atenderse el componente emocional, y que esto es especialmente relevante en el caso de los hombres. Además, como consecuencia del presente trabajo, podría destacarse la repercusión que estos hallazgos podrían tener en lo que respecta a la formación de dominio emocional de los emprendedores en el sector TIC (Salvador \& Mayoral, 2013). En este sentido, tal como recomiendan Baughn, Cao, Le, Lim \& Newpert (2006) y Wilson, Kickul \& Marlino (2007), convendría generar programas de intervención de auto-eficacia emprendedora. Ahora bien, según nuestros datos, en el sector tecnológico de la Argentina, cuando se organicen estos cursos de capacitación deberían atenderse varios aspectos: en primer lugar, si se trata de un hombre o una mujer; en segundo lugar, el grado de dominio emocional que muestran los emprendedores; y en tercer lugar, el grado de satisfacción vital, algo que adquiere un mayor peso cuando se trata de los hombres.

Este trabajo presenta algunas limitaciones a tener en cuenta en futuros estudios. En primer lugar, la investigación se ha desarrollado en un sector único -el tecnológico-. En segundo lugar, se ha utilizado un único momento de medida, por lo que convendría realizar estudios de carácter longitudinal. En tercer lugar, se ha utilizado como único instrumento de medida el cuestionario y como único informante el propio emprendedor. Pese a todo, como conclusión final de la presente investigación, convendría resaltar que conviene capacitar a los emprendedores con programas adaptados a sus exigencias, así pues se podría reducir las posibles brechas empresariales entre hombres y mujeres (Mueller \& Conway, 2013; Minniti, et al., 2005).

\section{Referencias}

Atienza, F., Pons, D., Balaguer, I. \& García-Merita, M. (2000). Propiedades psicométricas de la escala Satisfacción con la vida en adolescentes. En: Psicothema, 12(2): 314-319.

Bandura, A. (1987). Pensamiento y acción. Barcelona, España: Martínez Roca.

Bandura, A. (1997). Self-efficacy: The exercise of control. New York: Freeman.

Bandura, A. (1999). Auto-eficacia. Cómo enfrentamos los cambios de la sociedad actual. Bilbao, España: Desclée de Brouwer.

Baughn, C., Cao, J., Le, L, Lim, V. \& Newpert, K. (2006). Normative, social and cognitive predictors of entrepreuarial interest in China, Vietnam and Philippines. En: Journal of Developmental Entrepreneurship, 11(1): 57-77.

Chell, E., Haworth, J. \& Brearley, S. (1991). The entrepreneurial personality: concepts, cases and cathegories. London: Routledge.

Chen, C., Green, P. \& Crick, A. (1998). Does entrepreneurial selfefficacy distinguish entrepreneurs from managers? En: Journal of Business Venturing, 13: 295-316. 
Cohen, J. (2003). La inteligencia emocional en el aula. Argentina: Troquel.

De Noble, A., Jung, D. \& Ehrlich, S. (1999). Entrepreneurial selfefficacy: the development of a measure and its relationship to entrepreneurial actions. Trabajo presentado al Comunicación presentada en el Frontiers of Entrepreneurship Research, Waltham.

Díaz, J. \& Sánchez, M. (2002). Relaciones entre estilos de personalidad y satisfacción auto-percibida en diferentes áreas vitales. En: Psicothema, 14: 100-105.

Diener, E. (1984). Subjetive well-being. En: Psychological Bulletin, 95: 542- 575.

Diener, E. (1994). El bienestar subjetivo. Intervención psicosocial. En: Revista sobre igualdad y calidad de vida, 3(8): 67-113.

Diener, E., Emmons, R., Larsen, A. \& Griffin, R. (1985). The satisfactions with Life Scale. En: Journal of Personality Assessment, 49 (1): 71-75.

Diener, E., Suh, E., Lucas, R. \& Smith, H. (1999). Subjetive wellbeing: three decades of progress. En: Psychological Bulletin, 125 (2): 276-302.

Fernández-Berrocal, P. \& Extremera, L. (2006). La investigación de la inteligencia emocional en España. En: Ansiedad y Estrés, 12(2/3): 139-153.

Fernández-Berrocal, P., Extremera, N. \& Ramos, N. (2004). Validity and realiability of the spanish modified version of the trait meta- mood scale. En: Psychological Reports, 94: 751-755.

García, M. (2002). El bienestar subjetivo. En: Escritos de Psicología, 6: $18-39$.

García-Viniegras, C. \& González, I. (2000). La categoría bienestar psicológico, su relación con otras categorías sociales. En: Revista Cubana de Medicina Integral, 16 (6): 586-592.

Goleman, D. (1995). Inteligencia emocional. Barcelona: Kairós.

Heath, D. (1991). Fulfilling lives: paths to maturity and success. San Francisco: Jossey-Bass.

Kantis, H. Federico, J. \& Drucaroff, S. (2005) Clusters y nuevos polos emprendedores intensivos en conocimiento en Argentina. Buenos Aires. Disponible en: http://funcex.vgnet.com.br/material/ redemercosul bibliografia/biblioteca/ESTUDOS ARGENTINA/ ARG 50.pdf

Kantis, $\bar{H}$., Angelelli P. \& Moori Koenig, V. (2004) Desarrollo emprendedor: América Latina y la experiencia internacional. Ediciones Fundes. Banco Interamericano de desarrollo (BID). Disponible en: http:/www.fundes.org/uploaded/content/publicacione/1572456652.pdf

Kickul, J., Gundry, L., Barbosa, S. \& Whitcanack, L. (2009). Intuition versus analysis? Testing differential models of cognitive style on entrepreneurial self-effciacy and the new venture creation process. En: Entrepreneurship theory and practice, March: 439-453.

Liberalesso, A. (2002). Bienestar subjetivo en la vida adulta y la vejez: hacia una psicología positiva en América Latina. En: Revista Latinoamericana de Psicología, 34 (1-2): 55-74.

Markman, G., Baron R. \& Balkin, D. (2005). Are perseverance and self-efficacy costless? Assessing entrepreneurs' regretful thinking. En: Journal of Organizational Behavior, 26(1): 1-19.

Mayer, J. \& Salovey, P. (1997). What is emotional intelligence? En: Salovey, P. \& Sluyeter, D. (eds.). Emotional development and emotional intelligence: educational implications: 3-31. New York: Basic Books.

Mayer, J. \& Salovey, P. (1997). What is emotional intelligence? En: Salovey, P. \& Sluyter, D. (eds). Emotional development and emotional intelligence: implications for educators: 3-31. Nueva York: Basic Books.
Minniti, M., Arenius, P. \& Langowitz, N. (2005). GEM 2004 Report on Women and Entrepreneurship. Babson Park, MA: Global Entrepreneurship Monitor Program and Center for Women's Leadership at Babson College, Babson College.

Moriano, J., Palací, F. \& Morales, J. (2006). Adaptación y validación en España de la escala de autoeficacia emprendedora. En: Revista de Psicología Social, 21(1): 35-50.

Moyano, E. \& Ramos, N. (2007). Bienestar subjetivo: midiendo satisfacción vital, felicidad y salud en población chilena de la región Maule. En: Revista Universum, 22(2).

Mueller, S. \& Conway, M. (2013). A cross cultural study of genderrole orientation and entrepreneurial self-efficacy. En: Int. Entrep. Manag. Journal, 9: 1-20.

Pavot, W., Diener, E., Colvin, C. \& Sandwik, E. (1991). Future validation of the satisfaction with life scale: evidence for the cross-method convergence of well-being. En: Social Indicators Research, 28: 1-20.

Rego, A. \& Fernández, C. (2005). Inteligencia emocional: desarrollo y validación de un instrumento de medida. En: Revista Interamericana de Psicología, 39(1): 23-38.

Salovey, P. \& Mayer, J. (1990). Emotional intelligence. En: Imagination, Cognition and Personality, 9: 185-211.

Salovey, P., Mayer, J., Goleman, S., Turvey, C. \& Palfai, T. (1995). Emotional attention, clarity, and repair: exploring emotional intelligence using the trait meta-mood scale. En: Pennebaker, J. (ed.) Emotion, disclosure, \& health: 125-151. Washington, DC: American Psychological Association.

Salvador, C. (2010). Análisis transcultural de la inteligencia emocional. Universidad de Almería: Servicio de Publicaciones.

Salvador, C. (2012a). Influence of emotional intelligence in selfconcept. En: International Journal of Learning \& Development, 2(1).

Salvador, C. (2012b). How influence the new technologies in the emotional intelligence and communication of higher education student. En: Di Fabio, A. (ed.) Emotional intelligence: new perspectives and applications.

Salvador, C. \& Mayoral, L. (2013). Elementos determinantes de la satisfacción vital en el dominio emocional de las emprendedoras argentinas del sector TIC. Congreso Latino Iberoamericano de Gestión Tecnológica, Altec 2013, Porto - Portugal. Disponible en: http://www.altec2013.org/programme_pdf/118.pdf

Salvador, C. \& Morales, J. (2009). Fundamentos psicológicos de la autoeficacia emprendedora en jóvenes mexicanos. En: Alternativas en Psicología, 14 (20): 35-47.

Salvador, C., Mayoral, L. \& Errandoroso, F. (2012). Autoeficacia emprendedora en el sector de las TIC argentino: papel de la inteligencia emocional y la satisfacción vital. III Congreso de Gestión Tecnológica e Innovación - COGESTEC 2012, Medellín - Colombia.

Scherer, R., Brodzinski, J. \& Wiebe, F. (1990). Entrepreneur career selection and gender: a socialization approach. En: Journal of Small Business Management, Abril: 37-44

Shin, D. \& Johnson, D. (1978). Avowed happiness as an overall assessment of the quality of life. En: Social Indicators Research, $5 ; 475-492$.

Wilson, F., Kickul, J. \& Marlino, D. (2007). Gener, entreprenueurial self-efficacy, and entrepreneurial career intentions: implications for entrepreneurship education. En: Entrepreneurship Theory \& Practice, 31(3): 387-406. 